\title{
Higher perceived stress and poorer health reflected in elevated cortisol concentrations measured in extracts of hair from middle-aged healthy women
}

Åshild Faresjö ${ }^{1 *}$, Miriam Jullander ${ }^{1}$, Sara Götmalm ${ }^{1}$ and Elvar Theodorsson ${ }^{2,3}$

\begin{abstract}
Background: The prevalence of mental strain and stress has increased in modern societies, resulting in increased public health problems. Stress can be measured either by biomarkers or by self-reports. A new biomarker that measures long-term biological stress is cortisol measured in timed hair extracts. Hair grows at approximately $1 \mathrm{~cm}$ per month, and retrospectively reflects average stress levels. However, the plausible relationship between perceived stress and self-reported health and this novel biomarker is yet not firmly established. The objective of this study was to investigate the possible relationship between perceived stress, self-reported health, and cortisol in hair extracts in healthy middle-aged women from two different occupations.
\end{abstract}

Method: A cross-sectional study was conducted in 112 middle-aged women working as nurses or librarians in a county in southeast Sweden. The women were invited to fill in a questionnaire covering stress, health, and life situation. The questionnaire included questions on health and disease symptoms, the Perceived Stress Scale (PSS), and the Hospital Anxiety and Depression (HAD) scale. A piece of hair was cut from the vertex posterior area of the head an analysed by a competitive radioimmunoassay method.

Results: Middle-aged women who reported high perceived stress $(p=0.031)$ or lower health $(p=0.029)$, or had signs of depressiveness $(p=0.016)$ had significantly higher cortisol concentrations adjusted for age. There were no significant differences in cortisol in hair concentrations or perceived stress between nurses and librarians. Two women with extremely high cortisol concentrations were considered as outliers, but during the interview at follow-up they reported experiences of serious life events in their work or social life during the retrospective time of the sample taken for cortisol measurement.

Conclusions: Higher cortisol concentrations measured in the hair of healthy and working middle-aged women were associated with higher perceived stress and generally poorer health and with depressiveness. These findings lend support to the general applicability of cortisol measured in hair extracts as a biomarker in population-based epidemiological studies.

Keywords: Biomarker, Biological stress, Cortisol, HAD, Perceived health, Perceived stress

\footnotetext{
* Correspondence: ashild.olsen.faresjo@liu.se

'Division of Community Medicine, Department of Medicine and Health

Sciences, Faculty of Health Sciences, Linköping University, SE-581 83

Linköping, Sweden

Full list of author information is available at the end of the article
} 


\section{Background}

Stress is a frequent cause of work-related complaints in modern society and increased mental strain and stress are important determinants of public health problems (Wilkinson and Marmot 2003). Stress can be measured by biomarkers and/or perceived in responses to questionnaires. The hormone cortisol is commonly measured in saliva, blood, or urine. A drawback of these methods is that they do not reflect long-term stress exposure, only momentary stress. Cortisol measured in saliva and blood reflects current circulating cortisol levels, whilst cortisol measured in collections of urine reflects average cortisol concentrations up to 24 hours (Stalder and Kirschbaum 2012). An innovation for measuring long-term plasma cortisol concentrations is the assessment of cortisol in extracts from hair (Kirschbaum et al. 2006, Koren et al. 2002, Raul et al. 2004, Mayer and Novak 2012, Staufenbiel et al. 2013). Cortisol is a lipophilic compound assumed to be incorporated into hair after diffusion from blood plasma circulating in the capillaries that supply the hair roots as the hair grows. Sebum and sweat secretions might be other minor mechanisms of incorporation. Hair grows at approximately $1 \mathrm{~cm}$ per month but may slightly vary between individuals depending on race and sex (Wennig 2000). Thus, to obtain the average cortisol level reflecting the stress level during the last 3 months, a $3 \mathrm{~cm}$ long hair strand from the scalp outwards has to be cut from the vertex posterior. The cortisol levels in hair have found to be quite stable for at least 6 months and longer time periods (Davenport et al. 2006, Van Uum et al. 2008, Webb et al. 2010).

An increasing number of cross-sectional studies support the association between hair cortisol and different stressful conditions, but a more thorough understanding of the long-term effects of cortisol is warranted (Wester et al. 2014). A variety of contexts, exposures, situations and populations have been studied including unemployment (Dettenborn et al. 2010), shift work (Manenschijn et al. 2011), young adults (Karlén et al. 2011), living in a stressful community (Faresjö et al. 2013), and maternal influence on child (Karlén et al. 2013), and also predictors of hair cortisol concentrations in older adults (Feller et al. 2014), and cognitive performance for healthy older people (Pulopulos et al. 2014). Associations between cortisol concentrations and different physical and psychological symptoms as also been reported like; chronic pain (Van Uum et al. 2008), risk for heart infarction (Pereg et al. 2011), depression and mental illness (Dettenborn et al. 2012a, Steudte et al. 2013).

The individual's perception of stress or self-reported stress is not necessarily equivalent to stress measured by biomarkers. Self-reported stress can be measured by questionnaires and scales including the Perceived Stress Scale (PSS) questionnaire. PSS is a validated measurement of the extent to which situations in one's life are viewed as stressful (Cohen et al. 1983). However, there are several studies that did not find any or only weak associations between perceived stress and cortisol concentrations (Dettenborn et al. 2010, Karlén et al. 2011, Chan et al. 2014, Lambert et al. 2014), while this has been shown in some other studies (Kalra et al. 2007, Faresjö et al. 2013). These diverse results of the association between cortisol levels measured in hair and perceived stress, indicates that further studies are needed to get a more thoroughly picture of the potential association (Wells et al. 2014). Perceived health and it's potential association to levels of cortisol in hair has been analysed in a few studies (Karlén et al. 2011, Faresjö et al. 2013) accordingly this research question needs to be more explored. In general, the applicability of this method in epidemiological and public health studies engaging healthy people in different walks of life also needs to be more elaborated (Wosu et al. 2013).

\section{Study aims}

The aim was to investigate the possible relationship between perceived stress, self-reported health, and cortisol concentrations in hair amongst healthy and working middle-aged women from two different occupations.

\section{Methods}

\section{Participants and study design}

A cross-sectional study was conducted in women from two occupations in a county in southeast of Sweden. We decided to focus on two internationally quite common occupations; nurses and librarians. A hypothesis was that the workload for these two occupations might be different, with heavier workload and higher daily stress exposure on nurses than librarians. However, there are also similarities between these two occupations; women dominate both of them, they have about the same level of educational demand and also comparable salaries. The librarians worked at local Community libraries or at the University library. The nurses worked at the University Hospital employed by the County Council. Only nurses with ordinary day shifts (not nights) were recruited to participate in the study. General inclusion criteria were that the women should have hair of at least $3 \mathrm{~cm}$ in length. The sampling procedure in this study was convenience sampling, aimed to recruit at least have 50 persons from each occupation. Initially, a total of $\mathrm{N}=112$ women were recruited. Two participants had extremely high cortisol levels that distinctly stood out from the rest of the data since their cortisol levels was way over 2 SD from the mean, they were considered as outliers and omitted from the further statistical analyses. However, both of these two women were personally contacted and interviewed by telephone. The study thus rendered a total sample of $\mathrm{n}=110$ women, 
including $\mathrm{n}=58$ nurses and $\mathrm{n}=52$ library staff with a mean age of $45.8( \pm 11.7)$ years. It was unfortunately difficult to estimate the dropout rate, since all the women employed at each hospital clinic or library were offered to voluntarily participate by an open invitation. Of the contacted persons, some informed that they had no time to participate, while others declined to participate due to cosmetic reasons (recent hair cut). All participating women gave their informed and written consent to participate in the study.

\section{Questionnaire}

The participants were asked to answer a questionnaire including demographic variables such as age and occupation, and also possible confounders such as; tobacco use, longstanding medications, exercise habits, and if the respondent had colored or dyed/permed hair. Measuring tobacco smoking was a simple question whether the respondent at present or ever in the past had been a daily smoker. For medication, a question was asked whether the respondent at present used any long-standing medication in general and specifically whether the respondent at present used any medications including ointments, spray or tablets containing glucocorticoids (even specifying the most commonly used brands). Experiences of serious life events during the last three months including divorces, death of close relative, were registered. Furthermore, there were some questions about disease symptoms that may indicate stress, for instance, sleeping problems and stomach pain. All 14 items from the Hospital Anxiety and Depression (HAD) scale were included in the questionnaire (Zigmond and Snaith 1983). The scale is divided into two parts, anxiety (HADA) and depression (HADD), both consisting of seven items each. The 10item version of the Perceived Stress Scale (PSS) was also included in the questionnaire (Cohen et al. 1983, Cohen and Williamson 1988). A version of the PSS translated into Swedish language was used (Eskin and Parr 1996). The questionnaire including all these indicators has previously been applied in other studies from our research group (Karlén et al. 2011, Faresjö et al. 2013).

\section{Analysis of cortisol in hair}

Cortisol concentrations were expressed as $\mathrm{pg} / \mathrm{mg}$ with a method developed in-house using a competitive radioimmunoassay in methanol extracts of pulverised hair. An approximately $3 \mathrm{~mm}$ thick hair and $3 \mathrm{~cm}$ long sample was cut close to the scalp from the posterior vertex area of the head. Hair cortisol concentrations in the $3 \mathrm{~cm}$ hair segments reflect the cumulative cortisol secretion over the previous 3-month period, based on an assumption of an average hair growth rate of $1 \mathrm{~cm}$ per month (Wennig 2000). The hair samples analysed in this study weighted between $5 \mathrm{mg}$ and $6 \mathrm{mg}$. In the laboratory, each sample was cut finely, put into a $2 \mathrm{~mL}$ QiaGenRB sample tube together with a $0.5 \mathrm{~mm}$ QiaGen stainless steel bead, and weighed on a Sartorius MC 210p microscale. The samples were put in specially made aluminium cylinders accommodating five $2 \mathrm{~mL}$ Eppendorf tubes and frozen in liquid nitrogen for 2 minutes. This was followed by mincing in a Retch Tissue Lyser II at $23 \mathrm{~Hz}$ for 2 minutes to produce a fine hair powder. The cortisol was extracted by adding $1 \mathrm{~mL}$ of methanol (Chromasolv, Sigma-Aldrich) to each tube and placing the tubes in a metal holder on a plate with a 5-degree inclination on a horizontal shaker at room temperature, keeping the steel beads in constant gentle motion within the tubes for a minimum of 10 hours. Finally, the tubes were centrifuged for 1 minute at 13 $000 \mathrm{rpm}$ at $+4^{\circ} \mathrm{C}$ in a microcentrifuge, Thermo Scientific Heraus, Pico ${ }^{\text {tm }} \&$ Fresco $^{\text {tm }} 17 / 21$, and $800 \mu \mathrm{L}$ of the supernatant were moved to another plastic sample tube for lyophilisation in a SpeedVac Plus SC210A (Savant) using an Edwards XDS 5 vacuum pump for at least 2 hours. The samples were dissolved in radioimmunoassay buffer and analysed as described by Morelius et al. (2004).

Hair samples of 3-10 mg are needed to maintain a total inter-assay coefficient of variation below $8 \%$ for hair extraction and measurement of cortisol by the radioimmunoassay. The intra-assay coefficient of variation for the radioimmunoassay itself was $7 \%$ at $10 \mathrm{nmol} / \mathrm{L}$. The antiserum cross-reacts $137 \%$ with $5 \alpha$-dihydroxycortisol, $35.9 \%$ with 21-deoxycortisol, and $35.9 \%$ with prednisolone, but less than $1 \%$ with endogenous steroids.

\section{Statistical analysis and ethical approval}

Statistical analyses were performed using the Statistical Package for the Social Sciences (SPSS ver. 21). The cortisol concentrations were successfully log-transformed to a normal distribution before computing inferential statistics, since their distributions was skewed. The median and standard deviation were used as measures of central tendency and variation, respectively. The Pearson's correlation coefficient was used as measure of correlation. The bivariate correlations for perceived stress, self-reported health, depressiveness and cortisol levels were adjusted for age in multivariate linear regressions, $p$-values were considered significant if $\mathrm{p}<0.05$. The project was approved by the Research Ethics Committee at the Faculty of Health Sciences, Linköping University, Sweden, Dnr 2012/43-31.

\section{Results}

The median cortisol concentrations for the studied middle-aged women were $34.9 \mathrm{pg} / \mathrm{mg}$ with an interquartile range of 55.0 (CI 95\% 36.7 to 63.8). There was a significant correlation between self-reported health and cortisol levels. The Perceived Stress Scale (PSS) as well as indications of depressiveness derived from the Hospital Anxiety and scale (HADD) were also significantly associated with 
cortisol levels, see Table 1 and Figures 1, 2 and 3. All these three health outcomes remained significantly associated with cortisol concentrations after adjustment for age in multiple linear regressions, as shown in Table 1. Other measured health outcomes and sociodemographic factors did not show any significant associations with hair cortisol concentrations, including sleeping problems, stomach problems and longstanding illness. None of the potential confounders like; experience of serious life events, smoking, dyed/permed hair, exercise habits or use of ointments with cortisone, were significantly correlated with cortisol concentrations, see Table 1 . Nor was age significantly correlated with cortisol concentrations.

The comparison of the two occupations (nurses and librarians) reviled that there were no significant difference $(\mathrm{p}=0.79)$ adjusted for age of their cortisol concentrations in hair. There were neither any significant differences $(\mathrm{p}=$ $0.32)$ in perceived stress scale or HAD-depression $(\mathrm{p}=0.13)$ or HAD-anxiety $(\mathrm{p}=0.17)$ between nurses and librarians (adjusted for age) in this study. However, nurses reported significantly $(\mathrm{p}=0.04)$ better perceived health, adjusted for age, than librarians.

Table 1 Associations between different sociodemographic factors, health indicators and cortisol concentrations

\begin{tabular}{|c|c|c|c|c|c|c|c|}
\hline \multirow{3}{*}{$\begin{array}{l}\text { Health indicators } \\
\text { Self-reported health }(\min / \max : 2 / 10)\end{array}$} & \multicolumn{5}{|c|}{ Univariate associations } & \multicolumn{2}{|c|}{ Multivariate associations $s^{5}$} \\
\hline & \multirow{2}{*}{$\begin{array}{l}\mathbf{n}(\%) \\
110\end{array}$} & \multicolumn{2}{|c|}{ Cortisol pg/mg mean s.d. } & \multirow{2}{*}{$\begin{array}{l}\mathbf{r} \\
-0.21\end{array}$} & \multirow{2}{*}{$\begin{array}{l}\text { p-value } \\
0.027^{\# \#)}\end{array}$} & \multirow{2}{*}{$\begin{array}{l}\text { Beta } \\
-0.21\end{array}$} & \multirow{2}{*}{$\begin{array}{l}\text { p-value } \\
0.029\end{array}$} \\
\hline & & - & - & & & & \\
\hline Perceived Stress Scale (PSS) (min/max: 2/33) & 110 & - & - & 0.20 & $0.041^{\# \#)}$ & 0.21 & 0.031 \\
\hline HAD-depression (min/max: 0/10 & 110 & - & - & 0.23 & $0.014^{\# \#)}$ & 0.23 & 0.016 \\
\hline HAD-anxiety (min/max: 0/15) & 110 & - & - & 0.13 & $0.185^{\# \#)}$ & 0.15 & 0.131 \\
\hline Age (min/max: 22/66) & 110 & & & 0.09 & $0.36^{\# \#)}$ & - & - \\
\hline Serious life events & & & & 0.12 & $0.231^{\#)}$ & - & - \\
\hline Yes & $23(21)$ & 65.1 & 54.7 & & & & \\
\hline No & $87(79)$ & 49.4 & 55.9 & & & & \\
\hline \multicolumn{8}{|l|}{ Smoking habits } \\
\hline Smoker & $12(11)$ & 46.8 & 31.8 & 0.04 & 0.704 & - & - \\
\hline Non-smoker & $98(89)$ & 53.4 & 58.1 & & & & \\
\hline Dyed/permed hair & & & & & & - & - \\
\hline Yes & $54(49)$ & 47.5 & 45.6 & 0.09 & 0.344 & & \\
\hline No & $56(51)$ & 57.6 & 64.2 & & & & \\
\hline Exercise habits & & & & 0.18 & 0.184 & - & - \\
\hline Never & $14(13)$ & 75.9 & 101.4 & & & & \\
\hline Sometimes & $26(24)$ & 42.0 & 43.6 & & & & \\
\hline Regularly & $70(63)$ & 52.0 & 46.3 & & & & \\
\hline Ointments with cortisone & & & & 0.08 & 0.408 & - & - \\
\hline Yes & $9(8)$ & 37.1 & 24.3 & & & & \\
\hline No & $101(92)$ & 50.4 & 47.4 & & & & \\
\hline Longstanding illness & & & & 0.05 & $0.633^{\#)}$ & - & - \\
\hline Yes & $22(20)$ & 47.5 & 45.6 & & & & \\
\hline No & $88(80)$ & 53.9 & 58.2 & & & & \\
\hline Sleep disturbances & & & & 0.05 & $0.579^{\#)}$ & - & - \\
\hline Yes & $73(66)$ & 54.8 & 58.4 & & & & \\
\hline No & $37(34)$ & 48.5 & 50.8 & & & & \\
\hline Stomach pain & & & & 0.10 & $0.290^{\#)}$ & - & - \\
\hline Yes & $35(32)$ & 60.9 & 48.6 & & & & \\
\hline No & $75(68)$ & 48.8 & 58.8 & & & & \\
\hline
\end{tabular}

\#\#)Pearson correlation and cortisol concentrations.

\#) ANOVA and cortisol concentrations.

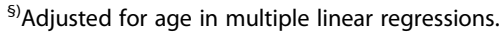




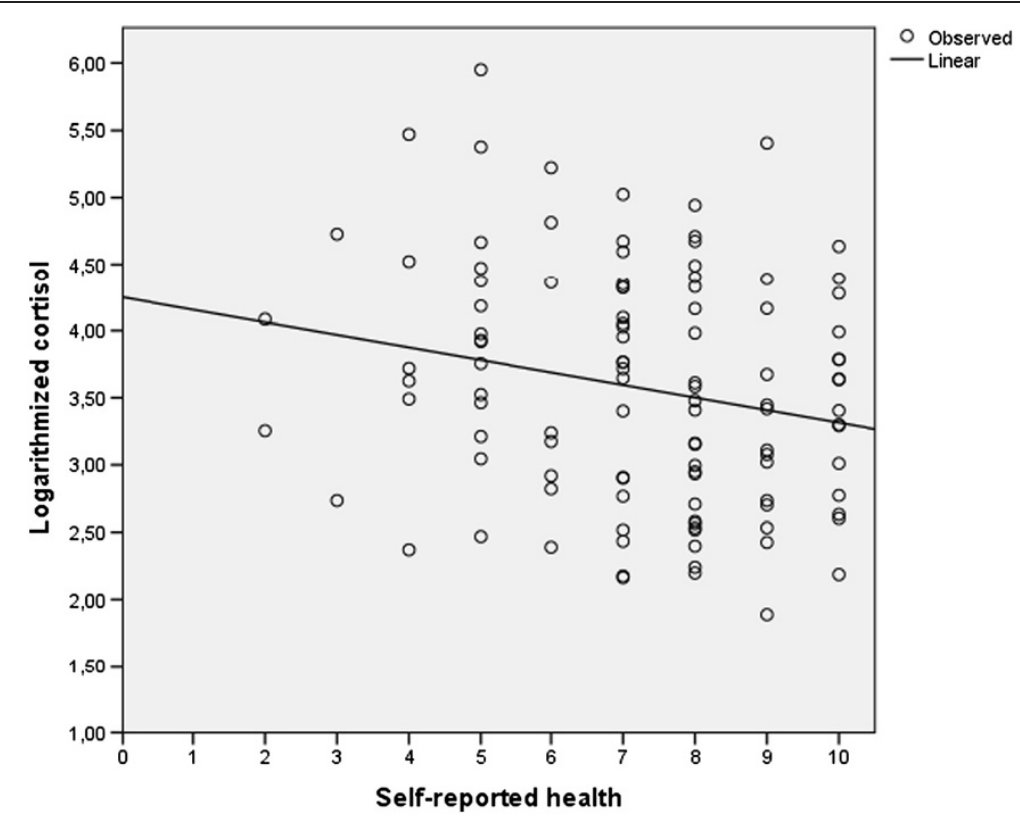

Figure 1 Associations between self-reported health and log-transformed cortisol concentrations (pg/mg), including a regression line.

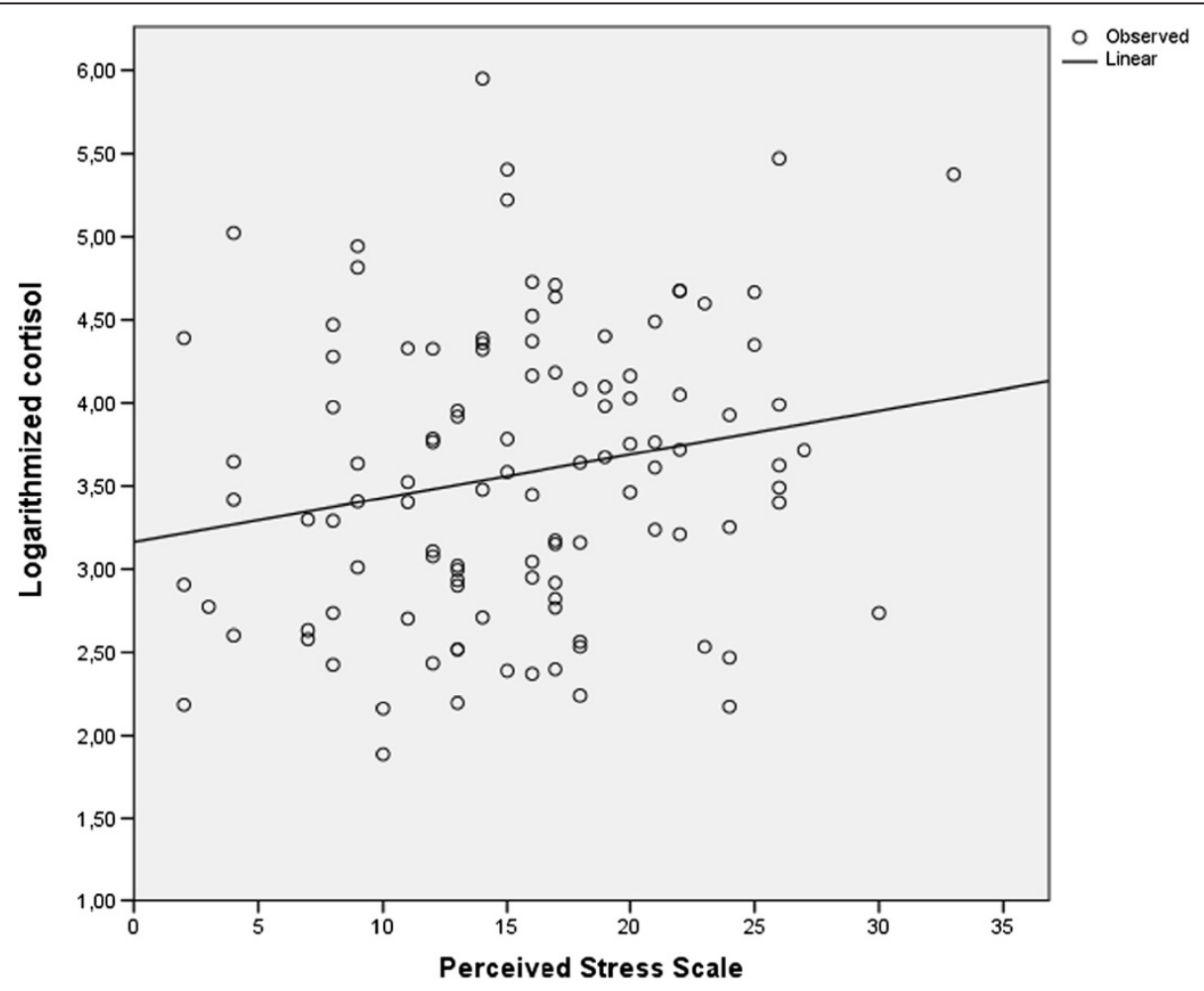

Figure 2 Associations between perceived stress and log-transformed cortisol concentrations (pg/mg), including a regression line. 


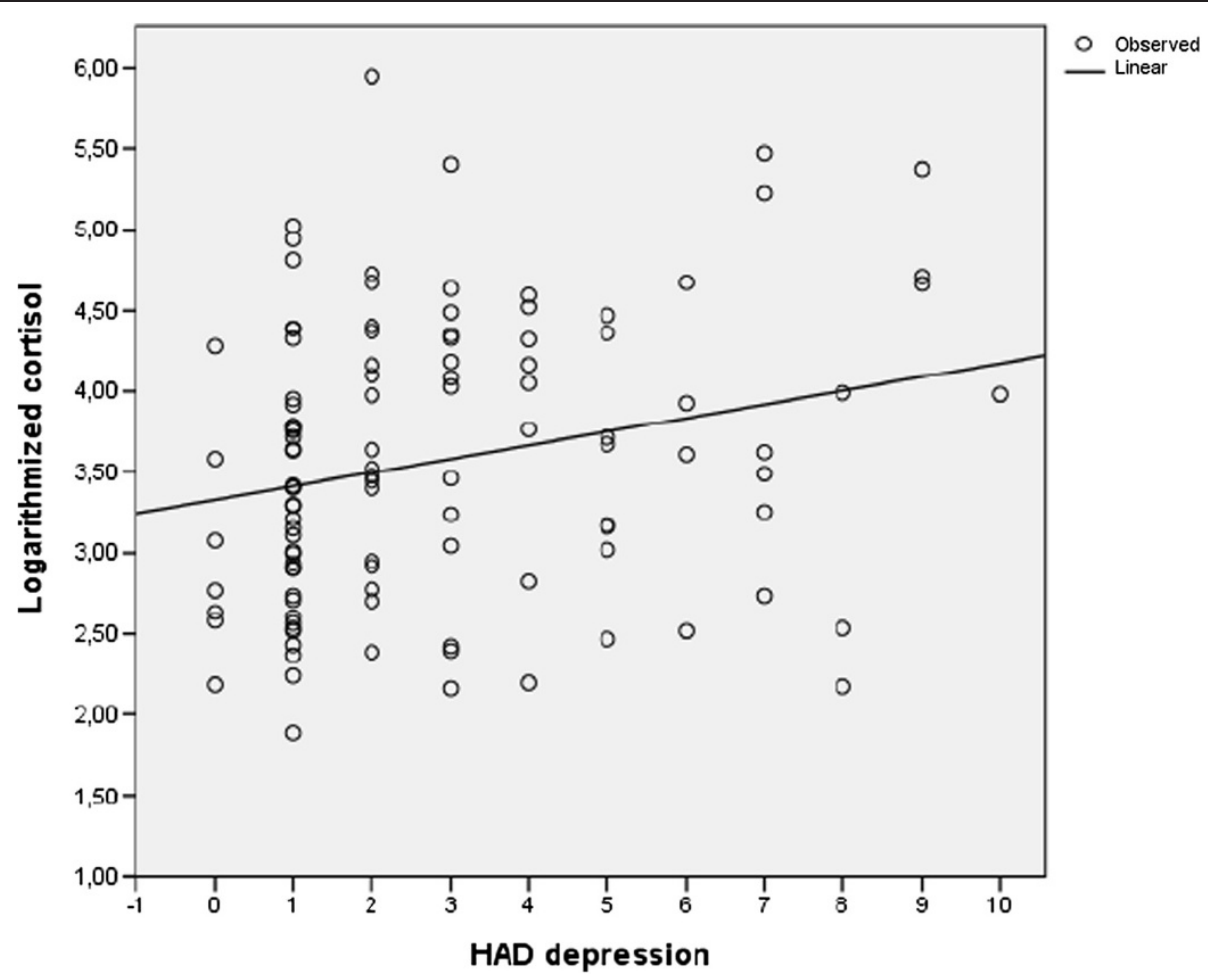

Figure 3 Associations between the Hospital Anxiety and Depression (HAD) scale HADD (depression) and log-transformed cortisol concentrations $(\mathrm{pg} / \mathrm{mg})$, including a regression line.

For the two persons considered as outliers in the study, special effort was made to obtain complementary information besides the questionnaire. Therefore, they were both interviewed by telephone. We hereby present them as 'case reports' within this study. Person A (a librarian aged 53 years) had a cortisol concentration of $16300 \mathrm{pg} / \mathrm{mg}$. This person reported that during the time period for the cortisol measurement, she had been on sick leave and was antidepressants since she had been exposed to severe violence at her work. Her individual values from the questionnaire was concerning PSS $=21 \mathrm{p} ; \mathrm{HADA}=13 \mathrm{p} ; \mathrm{HADD}=$ $6 \mathrm{p}$; self-reported health $=3$ ). Person $B$ (a librarian aged 33 years) had a cortisol concentration of $1679 \mathrm{pg} / \mathrm{mg}$. This person reported a stressful personal life situation due to a serious life event that had occurred during the months during which hair for the cortisol measurements had grown. Her individual values from the questionnaire was concerning PSS $=25$; HADA $=15$; $H A D D=11$; Selfreported Health $=2$. The values for these two outliers could be compared with the mean scores for the overall group that was for PSS $=15.5 \mathrm{p}$ (s.d. 6.5), $\mathrm{HADA}=6.6 \mathrm{p}$ (s.d. 3.4), HADD = 2.7p (s.d. 2.4) and for Self-reported Health $=7.25$ (s.d. 1.9).

\section{Discussion}

The main findings in this study was that healthy middleaged women reporting high perceived stress and poor health as well as those with signs of depressiveness had elevated cortisol concentrations in extracts from hair. The association between perceived stress and cortisol concentrations in hair extracts found is of special interest, since the results of previous similar reports are mixed (Dettenborn et al. 2010, Karlén et al. 2011, Lambert et al. 2014). Some previous studies like Kalra et al. (2007) showed that hair cortisol concentrations in pregnant women correlated positively and significantly with measures of perceived stress. A study comparing young adults in Greece and Sweden also found an association between high cortisol levels and PSS (Faresjö et al. 2013), while others have not found this association (Karlén et al. 2011, Chan et al. 2014). These diverse findings of this association indicates that further studies are needed to get a more thoroughly picture of the potential association (Wells et al. 2014).

The indicator of self-reported general health has scarcely been included in previous studies of cortisol measurements in hair. In the few available studies were this variable was analysed like; Karlén et al. 2011, Faresjö et al. 2013, and Feller et al. 2014, a somewhat mixed result were found, also indicating that further studies of this association is warranted. Symptoms of depressiveness measured with HADD in this study were associated with elevated cortisol concentrations. Some studies reported that depressed individuals tend to have higher 
cortisol concentrations compared with controls and that stress may lead to depression, while other studies could not find a clear connection between high hair cortisol concentrations and depressive symptoms (Dowlati et al. 2010, Dettenborn et al. 2012a).

In this study we did not find any significant difference in hair cortisol levels or perceived stress between nurses and librarians. Possible differences in workload and daily stress exposure between these two occupations might not be of that magnitude that it's depositing tracks that could be measured as biologically or perceived stress. Most previous research on cortisol in hair has focused on specific groups of individuals, certain stress exposures or patients with a variety of diseases, rather than on cohorts of regular and healthy people. Previous studies in this research area have mainly been cross-sectional. To further elucidate the mixed findings from previous studies, prospective studies are warranted.

Notably the associations between perceived stress, self-reported health, depressiveness, and cortisol concentrations were statistically significant, but in terms of correlations not so strong. Each of these three indicators explained only to a limited extent (around 5\%) the variability of the cortisol concentrations. However, it is not uncommon that even established associations between different social or psychosocial factors and health outcomes only explain a minimal magnitude of the variation (Abelson 1985). Care is warranted when interpreting individual cortisol concentrations despite the fact that the extracts from hair reflect cortisol built into the hair during months. We still do not know where in a cycle of periods of high or low stress exposure an individual is during the time period hair was collected for measuring cortisol concentrations. For the time being crosssectional measurements of cortisol in hair should therefore mainly be used when comparing groups. A question is how well the concordance is for humans between perceived stress, perceived health and the actual individual activity of the HPA-axis. This is a general research question that could be tackled in forthcoming studies.

In the present study, we did not found any associations with cortisol levels from the potential confounders that we measured in our study. We controlled for coloured or permed hair that might be a confounder for cortisol levels, but no such association was found in our study, which is in accordance with the results by Karlén et al. (2011). In contrast, Sauvé et al. (2007) found lower hair cortisol levels in dyed hair. We did neither found any correlations between cortisol levels and other potential confounders like; pharmaceutical (cortisone) consumption, experiences of serious life events, level of exercise or smoking. In some previous studies associations was found between experience of serious life events and cortisol levels (Karlén et al. 2011) but another study failed to reveal consistent associations between different stress-related psychosocial measures and hair cortisol (Stalder et al. 2012b). For substances used like alcohol and smoking and cortisol levels, the association is not clear (Wells et al. 2014, Wosu et al. 2013). Some studies have found that use of chemicals in hair treatments like; bleaching, dyeing, straightening, or permanent waves might interfere with the cortisol concentrations (Sauvé et al. 2007, Manenschijn et al. 2011), but other studies found no such effect (Dowlati et al. 2010, Stalder et al. 2012a). However, the use of hair spray, gel, and wax appear not to have any influence on hair cortisol levels (Manenschijn et al. 2011). Other health indicators measured in this study like; chronic diseases, sleep disturbances, and stomach pain, were not significantly associated with cortisol concentrations.

The additional information gained from the case reports of the two persons considered as outliers in this study calls for special attention. To minimise the risk for bias due to methodological problems that might explain the high cortisol levels for these two participants, several measures were made. The procedure in our laboratory if outliers are detected is that their biological samples are replicated and analysed at two independent occasions, which was done for these two outliers, giving the same and stable results. Further, we ensured that these two persons had not used glucocorticoids recent months, which could be potential confounders possibly explaining these high cortisol values, that was neither the case. The research of cortisol concentrations in hair is still in it's initial stage. To our knowledge, no clinical standards or reference values have so far been presented, so the magnitude of the physiologically plausible cortisol in hair concentrations is unknown. In our present study, both women with extremely high cortisol levels had experienced severe life events either at work or in their social life during the retrospective period when the cortisol in hair was measured. About the same high magnitude of cortisol levels was found in another study where cortisol levels and stress were studied in young adults (Karlén et al. 2011). In that study two out of four outliers that was interviewed reported severe mental problems. This previous study as well as the present therefore raises the hypothesis that measurement of cortisol in hair might detect serious life events and severe mental problems that have strongly affected the cortisol concentrations in plasma of the affected individuals.

\section{Strengths and limitations}

In this study, the sample consists of working middle-aged nurses and librarians. These two occupations were deliberately chosen to get a wider variety of workload and daily stress exposure in two female-dominated occupations, this could possibly be a limitation. An alternative sampling could have been to only focus on one occupation and 
thereby achieve more homogeneity or working women in general. However, educational level and salary were about the same for both occupations, making them comparable in these aspects. Strength in this study is that these studied women were "common people" and not "clinical cases" with internationally quite well known occupations. A limitation is that they are not representative of the general population and also that the study design is cross-sectional and not longitudinal correlating symptoms and cortisol concentrations in hair in samples repeated over extended periods of time where each person is his/her own control. The participating women were all working at the time of data collection so they could be considered as a relatively healthy cohort of middle-aged women. However, factors like age and sex could not be excluded as confounding factors in studies of cortisol in hair (Dettenborn et al. 2012b, Feller et al. 2014). Our study only included women and age was adjusted for in the multivariate analysis. The sampling procedure used was a convenience sampling rather than a random sampling, which is a limitation in this study.

A strength with the questionnaire used is that both PSS and HAD are validated scales. The purpose of using these questionnaires was to measure stress level during the last 3 months, but the original scale of PSS is only validated for 1 month back in time, which might be a limitation in this study. The HAD scale only measures the anxiety and depressiveness levels 'right now' and not retrospectively. On the other hand, the presence of depressiveness or anxiety has a natural history that is backwards in time. The question concerning serious life events was only asked for events during the last 3 months, although events occurring earlier might have been very stressful and therefore could have affected the hair cortisol levels.

Another strength in this study was that the hair was cut into pieces as part of the analytical process in the laboratory to ensure that all analysed hair was from the latest three months. The possible uncertainty when extracting and measuring cortisol in hair samples has been minimized in this study since the applied method used has previously been tested in several previous studies (Karlén et al. 2011, Karlén et al. 2013, Faresjö et al. 2013). Compared with measuring cortisol in saliva, blood, and urine, measuring cortisol in hair has several advantages. Measuring cortisol in hair is a non-invasive method, it is possible to store hair at room temperature, and a non-professional can collect the hair sample (Raul et al. 2004). There is also strong evidence for a high level of intraindividual stability in hair cortisol concentrations (Stalder and Kirschbaum 2012).

\section{Conclusions}

In conclusion, higher cortisol levels measured in hair during the past months are associated with; higher perceived stress during the previous month, lower perceived health and also symptoms of depressiveness for healthy and working middle-aged women. There were no significant differences in cortisol concentrations or perceived stress between nurses and librarians. The associations found in this study between different health indicators and cortisol levels measured in hair lend some support to the general applicability of this method in populationbased epidemiological studies.

\section{Competing interests \\ The authors declare that they have no competing interests.}

\section{Authors' contributions}

$\AA \mathrm{F}, \mathrm{SG}, \mathrm{MJ}$ and $\mathrm{ET}$ participated in the study design and coordination and SG, MF completed the data collection. ET, ÅF developed the method. ÅF, SG, MJ, $\mathrm{ET}$, drafted the manuscript. All authors contributed to analysis and interpretation of data, and read and approved the final manuscript.

\section{Acknowledgements}

We thank all women who participated in this study. This study was partly supported by the Medical Program at the Faculty of Health Sciences, Linköping University, Sweden.

\section{Author details}

${ }^{1}$ Division of Community Medicine, Department of Medicine and Health Sciences, Faculty of Health Sciences, Linköping University, SE-581 83 Linköping, Sweden. ${ }^{2}$ Division of Microbiology and Molecular Medicine, Department of Clinical and Experimental Medicine, Faculty of Health Sciences, Linköping University, Linköping SE-581 83, Sweden. ${ }^{3}$ Department of Clinical Chemistry, Center for Diagnostics, County Council of Östergötland, SE-581 85 Linköping, Sweden.

Received: 23 March 2014 Accepted: 15 August 2014 Published: 31 August 2014

\section{References}

Abelson, R. (1985). A variance explanation paradox: when a little is a lot Psychological Bulletin, 97(1), 129-133.

Chan, J, Sauvé, B, Tokmakejian, S, Koren, G, \& van Uum, S. (2014). Measurement of cortisol and testosterone in hair of obese and non-obese human subjects. Current Opinion in Endocrinology, Diabetes, and Obesity, 21(4), 306-311.

Cohen, S, \& Williamson, GM. (1988). Perceived stress in a probability sample of the United States. In S Spacapan \& S Oskamp (Eds.), The Social Psychology of Health (pp. 31-67). Newbury Park, CA: Sage.

Cohen, S, Kamarck, T, \& Mermelstein, R. (1983). A global measure of perceived stress. Journal of Health and Social Behavior, 24(4), 385-396.

Davenport, MD, Tiefenbacher, S, Lutz, CK, Novak, MA, \& Meyer, JS. (2006). Analysis of endogenous cortisol concentrations in the hair of rhesus macaques. General and Comparative Endocrinology, 147, 255-261.

Dettenborn, L, Tietze, A, Bruckner, F, \& Kirschbaum, C. (2010). Higher cortisol content in hair among long-term unemployed individuals compared to controls. Psychoneuroendocrinology, 35(9), 1404-1409.

Dettenborn, L, Muhtz, C, Skoluda, N, Stalder, T, Steudte, S, Hinkelmann, K, Kirschbaum, C, \& Otte, C. (2012a). Introducing a novel method to assess cumulative steroid concentrations: Increased hair cortisol concentrations over 6 months in medicated patients with depression. Stress, 15(3), 348-353.

Dettenborn, L, Tietze, A, Kirschbaum, C, \& Stalder, T. (2012b). The assessment of cortisol in human hair: associations with sociodemographic variables and potential confounders. Stress, 15(6), 578-588.

Dowlati, Y, Herrmann, N, Swardfager, W, Thomson, S, Oh, PI, Van Uum, SH, Koren, G, \& Lanctôt, KL. (2010). Relationship between hair cortisol concentrations and depressive symptoms in patients with coronary artery disease. Neuropsychiatric Disease and Treatment, 6, 393-400.

Eskin, M, \& Parr, D. (1996). Introducing a Swedish Version of an Instrument Measuring Mental Stress: Report No 813. Stockholm: Department of Psychology, Stockholm University.

Faresjö, Å, Theodorsson, E, Chatziarzenis, M, Sapouna, V, Claesson, HP, Koppner, J, \& Faresjö, T. (2013). Higher perceived stress but lower cortisol levels found among young Greek adults living in a stressful social environment in 
comparison with Swedish young adults. PLoS One, 8(9), e73828. doi:10.1371/journal.pone.0073828. eCollection.

Feller, S, Vigl, M, Bergmann, MM, Boeing, H, Kirchbaum, C, \& Stalder, T. (2014). Predictors of hair cortisol concentrations in older adults. Psychoneuroendocrinology, 39, 132-140.

Kalra, S, Einarson, A, Karaskov, T, Van Uum, SH, \& Koren, G. (2007). The relationship between stress and hair cortisol in healthy pregnant women. Clinical and Investigative Medicine, 30(2), 103-107.

Karlén, J, Ludvigsson, J, Frostell, A, Theodorsson, E, \& Faresjö, T. (2011). Cortisol in hair measured in young adults - a biomarker of major life stressors? BMC Clinical Pathology, 11(1), 12. doi:10.1186/1472-6890-11-12.

Karlén, J, Frostell, A, Theodorsson, E, Faresjö, T, \& Ludvigsson, J. (2013). Maternal influence on child HPA axis: a prospective study of cortisol levels in hair. Journal of Pediatrics, 132(5), e1333-e1340. doi:10.1542/peds.2013-1178. Epub 2013 Oct 7

Kirschbaum, C, Tietze, A, Skoluda, N, \& Dettenborn, L. (2006). Hair as a retrospective calendar of cortisol production-increased cortisol incorporation into hair in the third trimester of pregnancy. Psychoneuroendocrinology, 34, 32-37.

Koren, L, Mokady, O, Karaskov, T, Klein, J, Koren, G, \& Geffen, E. (2002). A novel method using hair for determining hormonal levels in wildlife. Animal Behaviour, 63, 403-406.

Lambert, CA, Sanders, A, Wilder, RS, Slade, GD, Van Uum, S, Rusell, E, Koren, G, \& Maixner, W. (2014). Chronic HPA-axis response to stress in temporomandibular disorder. Journal of Dental Hygiene, 88(Suppl 1), 5-12.

Manenschijn, L, Koper, JW, Lamberts, SW, \& van Rossum, EF. (2011). Evaluation of a method to measure long term cortisol levels. Steroids, 76, 10-11.

Mayer, JS, \& Novak, MA. (2012). Minireview: hair cortisol: a novel biomarker of hypothalamic-pituitary-adrenocortical activity. Endocrinology, 153(9), 4120-4127.

Morelius, E, Nelson, N, \& Theodorsson, E. (2004). Salivary cortisol and administration of concentrated oral glucose in newborn infants: improved detection limit and smaller sample volumes without glucose interference. Scandinavian Journal of Clinical \& Laboratory Investigation, 64(2), 113-118.

Pereg, D, Gow, R, Mosseri, M, Lishner, M, Rieder, M, Van Uum, SH, \& Koren, G. (2011). Hair cortisol and the risk for acute myocardial infarction in adult men. Stress, 14(1), 73-81.

Pulopulos, MM, Hidalgo, V, Almela, M, Puig-Perez, S, Villada, C, \& Salvador, A. (2014). Hair cortisol and cognitive performance in healthy older people. Psychoneuroendocrinology, 44, 100-111.

Raul, JS, Cirimele, V, Ludes, B, \& Kintz, P. (2004). Detection of physiological concentrations of cortisol and cortisone in human hair. Clinical Biochemistry, 37(12), 1105-1111.

Sauvé, B, Koren, G, Walsh, G, Tokmakejian, S, \& Van Uum, SH. (2007). Measurement of cortisol in human hair as a biomarker of systemic exposure. Clinical and Investigative Medicine, 30(5), 183-191.

Stalder, T, \& Kirschbaum, C. (2012). Analysis of cortisol in hair - state of the art and future directions. Brain, Behavior, and Immunity, 26(7), 1019-1029.

Stalder, T, Steudte, S, Miller, R, Skoluda, N, Dettenborn, L, \& Kirschbaum, C. (2012a). Intraindividual stability of hair cortisol concentrations. Psychoneuroendocrinology, 37(5), 602-610.

Stalder, T, Steudte, S, Alexander, N, Miller, R, Gao, W, Dettenborn, L, \& Kirschbaum, C. (2012b). Cortisol in hair, body mass index and stress-related measures. Biological Psychology, 90, 218-223.

Staufenbiel, SM, Pennix, BW, Spijker, AT, Elzinga, BM, \& van Rossum, EF. (2013). Hair cortisol, stress exposure, and mental health in humans: a systematic review. Psychoneuroendocrinology, 38, 1220-1235.

Steudte, S, Kirschbaum, C, Gao, W, Alexander, N, Schonfeld, S, Hoyer, J, \& Stalder, T. (2013). Hair cortisol as a biomarker of traumatization in healthy individuals and posttraumatic stress disorder patients. Biological Psychiatry, $74(9), 639-646$

Van Uum, SH, Sauvé, B, Fraser, LA, Morley-Forster, P, Paul, TL, \& Koren, G. (2008). Elevated content of cortisol in hair of patients with severe chronic pain: a novel biomarker for stress. Stress, 11(6), 483-488.

Webb, E, Thomson, S, Nelson, A, White, C, Koren, G, Rieder, M, \& van Uum, SH. (2010). Assessing individual systemic stress through cortisol analysis of archaeological hair. Journal of Archaeological Science, 37, 807-812.

Wells, S, Tremblay, PF, Flynn, A, Rusell, E, Kennedy, J, Rehm, J, Van Uum, S, Koren, G, \& Graham, K. (2014). Associations of hair cortisol concentration with self-reported measures of stress and mental health-related factors in a pooled database of diverse community samples. Stress, 17(4), 334-342.
Wennig, R. (2000). Potential problems with the interpretation of hair analysis results. Forensic Science International, 107(1-3), 5-12.

Wester, VL, Lamberts, SW, \& van Rossum, EF. (2014). Advances in the assessment of cortisol exposure and sensitivity. Current Opinion in Endocrinology, Diabetes, and Obesity, 21(4), 306-311.

Wilkinson, R, \& Marmot, M. (2003). Social Determinants of Health: The Solid Facts (2nd ed.). Copenhagen: WHO Regional Office for Europe.

Wosu, AC, Valdimarsdòttir, U, Shields, AE, Williams, DR, \& Williams, MA. (2013). Correlates of cortisol in human hair, implications for epidemiologic studies on health effects of chronic stress. Annals of Epidemiology, 23, 797-811.

Zigmond, AS, \& Snaith, RP. (1983). The hospital anxiety and depression scale. Acta Psychiatrica Scandinavica, 67, 361-370.

\section{doi:10.1186/s40359-014-0030-7}

Cite this article as: Faresjö et al:: Higher perceived stress and poorer

health reflected in elevated cortisol concentrations measured in extracts of hair from middle-aged healthy women. BMC Psychology 2014 2:30.

\section{Submit your next manuscript to BioMed Central and take full advantage of:}

- Convenient online submission

- Thorough peer review

- No space constraints or color figure charges

- Immediate publication on acceptance

- Inclusion in PubMed, CAS, Scopus and Google Scholar

- Research which is freely available for redistribution 